\title{
Effect of shRNA-Mediated Knockdown EBF1 Gene Expression on the Proliferation of Lung Cancer Cell Line A549 In Vitro and In Vivo
}

\section{Lin WANG}

Department of Laboratory, Tianjin medical university cancer institute and hospital

\section{Ding Li}

Department of Laboratory, Tianjin medical university cancer institute and hospital

\section{Li REN ( $\nabla$ roland-li@163.com )}

Department of Laboratory, Tianjin medical university cancer institute and hospital

\section{Honglei FENG}

Department of Laboratory, Tianjin medical university cancer institute and hospital

\section{Research Article}

Keywords: Lung adenocarcinoma, EBF1, Proliferation

Posted Date: June 2nd, 2021

DOI: https://doi.org/10.21203/rs.3.rs-514722/v1

License: (c) (i) This work is licensed under a Creative Commons Attribution 4.0 International License. Read Full License 


\section{Abstract}

\section{Background}

The incidence of lung cancer is increasing year by year. The study on the proliferation and metastasis of lung adenocarcinoma cells is of positive significance to improve the prognosis of patients with lung adenocarcinoma, but there is still a lack of more effective treatment for the proliferation and metastasis of lung adenocarcinoma cells. Here we find that a lymphocyte lineage specific transcription factor,EBF1, is frequently expressed in human lung cancer tissues and affects the proliferation of tumor cells, Objective to explore the possible mechanism of affecting the proliferation of lung adenocarcinoma cells.

\section{Methods}

Immunohistochemistry and PCR were used to detect the expression of EBF1 in lung cancer tissues and lung cancer cell lines. According to the interference RNA (shRNA) sequence designed by our laboratory for $E B F 1$ as the target sequence and a random sequence as the negative control, the recombinant retrovirus was constructed and transfected into A549 cells, which were used as A549-shRNA-EBF1 and A549shRNA-control of experimental group and control group respectively; Knockdown of EBF1 gene was detected by PCR and Western blot. MTT and BrdU staining were used to detect the effect of EBF1-shRNA on the proliferation of A549 cells in vitro;flow cytometry was used to analyze the cell cycle of each group; subcutaneous inoculation of cells in axilla of nude mice was used to observe the effect of EBF1-shRNA on the tumorigenicity of A549 cells in nude mice; Western blot was used to detect the expression of CDK6, P21 and P27 proteins.

\section{Results}

EBF1 was not expressed in stromal cells of adjacent tissues and lung cancer tissues, but in nuclei of NSCLC and SCLC cancer cells. EBF1-shRNA knockdown EBF1 gene expression effectively; knockdown EBF1 gene expression can inhibit the proliferation of A549 cells in vitro and in vivo, and block the cell cycle of experimental group at G1 phase; after knockdown EBF1 gene expression, CDK6 protein expression in experimental group cells decreases, while P21 and P27 protein increase.

\section{Conclusions}

EBF1-shRNA can inhibit the proliferation of lung adenocarcinoma A549 in vitro and in vivo by blocking cell cycle in G1 phase, which involves the decrease of CDK6 expression and the up-regulation of P21 and P27 expression. This study will supplement the theory that heterotopic expression of hematogenous transcription factors in lung cancer affects tumor proliferation and discover new molecular targets for cancer therapy.

\section{Introduction}


Cell proliferation is an important section in tumorigenesis and development. We found that EBF1 (Early BCell Factor 1), a key transcription factor for hematopoietic system development, was up-regulated in lung cancer cells. Knockdown EBF1 expression in lung adenocarcinoma cell A549 could inhibit the proliferation of lung adenocarcinoma cells and inhibit the growth of tumors in vivo. Its mechanism may be related to its influence on cell cycle. This study will supplement the theory that heterotopic expression of hematogenous transcription factors in lung cancer affects tumor proliferation and discover new molecular targets for cancer therapy.

In recent years, the incidence of lung cancer has increased year by year, and the five-year survival rate is only $15 \%{ }^{[1]}$. About $85 \%$ of them are non-small cell lung cancer (NSCLC) ${ }^{[2]}$. Lung adenocarcinoma is a common type among women and non-smokers in NSCLC ${ }^{[3]}$. Tumor formation is often caused by mutations in genes, including activation of oncogenes and inactivation or deletion of tumor suppressor gene. For example, INK4a/ARF encodes two inhibitory proteins, p16INK4a and p14ARF ${ }^{[4]}$, respectively. INK4a/ARF locus is often absent in lung adenocarcinoma ${ }^{[5]}$. INK4a/ARF locus is located in the 1 st band of chromosome 9 short arm 2 and is a cyclin dependent kinase (CDK) inhibitor. P16INK4a and p14ARF are cell cycle regulators and play important roles in $\mathrm{Rb}$ and $\mathrm{p} 53$ pathways.

EBF1 gene is located in $5 q 34$ of human genome, which coding product EBF1 is a necessary transcription factor for B cell development and plays an important role in B cell differentiation and maturation ${ }^{[6]}$. It has been found that EBF1 is necessary for the proliferation, survival, maturation and signal transduction of pre-B cells and peripheral blood B cell subsets. EBF1 and E2A coordinate to regulate the transcription of $B$ cell-specific genes including VpreB (Pre-B-Lymphocyte) and mb-1[7],which encodes the Ig-alpha signaling component of the $B$ cell receptor.EBF1 promotes the expression of $B$ cell markers such as immunoglobulin by enhancing the transcriptional activity of DNA demethylation ${ }^{[8]}$.Some studies have shown that there is a deletion of EBF1 expression in childhood acute lymphoblastic leukemia ${ }^{[9,10]}$, the frequency of EBF1 deletions was higher in pediatric high-risk B-precursor ALL. Especially the EBFIPDGFRB fusion gene makes the differentiation of cancer cells stagnate at the stage of pre-B cell (due to the defect of EBFI function) and continuous proliferation (due to the disorder of PDGFRB kinase activity) [11]

Recently, Stone $\mathrm{J}$ found that EBF1 and ESR1 are associated with increased susceptibility to breast cancer ${ }^{[12]}$. It may inferred that EBF1 also plays an important role in the development of solid tumors, but the molecular mechanism of its function, especially the effect on lung cancer, is still unclear.

For this reason, we used immunohistochemical and western blot methods to identify the expression of EBF1 in lung cancer tissues and lung cancer cell lines. We designed hairpin EBF1 shRNA and constructed stable EBF1 shRNA to transfect A549 cells to knockdown EBF1 gene. The effects of EBF1 expression on the proliferation of lung cancer cells were examined by cell proliferation assay, cell cycle assay and in vivo animal experiments in mice,to explore the possible molecular mechanism of EBF1 involvement in 
lung cancer cell proliferation ,and investigate the expression of EBF1 in lung cancer cells and its biological significance.

\section{Materials And Methods}

\section{Cell Lines}

HBEC cells are normal human bronchial epithelium immortalized by hTERT and CDK4, and were a kind gift of Jerry Shay (UT Southwestern Medical Center, Dallas, TX). BEAS-2B cells were isolated from the normal human bronchial epithelium obtained from autopsy of non-cancerous individuals. BEAS-2B,H209, H1155, H441,A549 cells were from ATCC (American Type Culture Collection)and maintained in ATCC recommended media supplemented with $10 \%$ FBS(Fatal Bovine Serun), $100 \mathrm{U}$ of penicillin/ $\mathrm{ml}$, and $100 \mu \mathrm{g}$ of streptomycin/ml. All experiments were performed within 1 month after thawing early-passage cells.

\section{Immunohistological staining}

Lung cancer tissues were obtained from Tianjin Medical University Cancer Institute and Hospital in China. Samples were embedded in $4 \%$ paraformaldehyde of paraffin at $4^{\circ} \mathrm{C}$ overnight to fix . Formalin-fixed tissues should be cutted into $5 \mathrm{~mm}$ sections and deposited on glass slides. Tissues were rehydrated, blocked in $10 \%$ NGS(Normal Goat Serum) of PBS(Phosphate Buffered Saline), and sequentially incubated with specific primary antibody, EBF1 $\triangle S I G M A$ Sc-137065区 and with biotinylated secondary antibodies 囚Bioworld Technology $₫$ for immunohistochemistry.

\section{In vivo metastasis assay}

According to GFP expression $₫ A 549$ cells expressing empty vector or knockdown EBF1 were selected by cell sorting (FACS Vantage, BD Biosciences). $10^{7}$ cells for each group with 100 ul saline were injected under the skin of 8-week-old female nude mice. The tumor in situ was removed 3 weeks after cell inoculation. All animal procedures were approved by the animal care and use Committee of Tianjin Medical University, in accordance with the law and the National Laboratory Animal Care and maintenance guidelines.

\section{Western blot}

1/20 beta-mercaptoethanol was used to lyse cells in Laemmli sample buffer (Bio-Rad) on ice, to extract the total protein.The protein concentration was determined by ultramicro spectrophotometer Nanodrop 2000.Then the cell lysates were loaded and resolved by $10 \%$ SDS/PAGE (120 V, until the blue indicator runs to the edge of the gel, at room temperature.). Proteins were then transferred onto poly(vinylidene difluoride) (PVDF) membranes (100 V, 70min, on ice). Then the membranes were blocked with $5 \%$ milk in PBS added with Tween-20 (PBST) for $1 \mathrm{~h}$ at room temperature, and then incubated overnight at $4{ }^{\circ} \mathrm{C}$ with

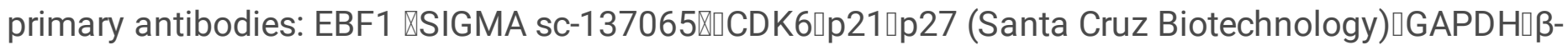
actin (BD Biosciences). The PVDF membrane was washed three times (10 min each time) with PBST and 
then incubated with HRP-conjugated secondary antibodies at room temperature for $1 \mathrm{~h}$. After extensive washing by PBST, the membrane was prepared by ECL method..

\section{MTT assay}

Collect logarithmic phase cells to a 96-well plate for MTT assays at a density of $3 \times 103$ cells/well overnight at $37^{\circ} \mathrm{C}$ with $5 \% \mathrm{CO} 2$. Each group has five multiple holes, which are continuously tested for 5 days. cells were incubated with $20 \mu \mathrm{l}$ of MTT solution at $5 \mathrm{mg} / \mathrm{ml}$ for $4 \mathrm{~h}$ at $37^{\circ} \mathrm{C}$ in a humidified CO2 incubator. The media were removed, and then $150 \mu \mathrm{l}$ dimethylsulphoxide (DMSO) was added into each well and incubated at room temperature for $15 \mathrm{~min}$. Finally, the absorbance was measured at $490 \mathrm{~nm}$ with a microplate reader

\section{Results}

\section{EBF1 is expressed in lung cancer cells}

To explore the role of EBF1 in human lung cancer,we using immunohistochemical (IHC) analyses examined clinical human lung cancer tissue and using Semiquantitative RT-PCR reactions examined various cancer cell lines for EBF1 expression. Normal lung epithelial cells in tumor-adjacent lung tissues and stromal cells in lung cancer tissues did not express EBF1. Both NSCLC and SCLC cells expressed EBF1, and all EBF1-positive lung cancer cells are all exhibited nuclear localization. (Fig 1 A)

To more closely assess the significance of EBF1 in human cancer,we further examined various cancer cell lines for EBF1 expression. Figure 1B showed that EBF1 expression increased in human lung cancer cell lines compare with normal human bronchial epithelial cells (HBEC cells and BEAS-2B cells)and several human lung cancer cell lines including A5490H4410H209 and H1155.

\section{EBF1 knockdown inhibit cell proliferation in A549 cells}

The result of RT-PCR demonstrated that the high expression of EBF1 in lung cancer cells, to explore the influence of EBF1 to lung cancer,we use shRNA downregulate EBF1 in A549 cells. It was found that the expression of EBF1 in A549 cells transfected with specific shRNA decreased significantly (Fig 2A).With BrdU experiment to detect the cell proliferation level, we found that knockdown EBF1 inhibit the proliferation of A549 cells compared with control group (Fig 2B). To analyse the cell viability ,we use the MTT assay in A549 cells. We found the growth rate was significantly slower in A549-shRNA-EBF1 cells than that of A549-shRNA-control cells on day 3 and later(Fig 2C). Similarly, the proliferation of H441 cells decreased $(P<0.05)$ and the growth rate was significantly slower $(P<0.05)$ compared with the control group (Fig. 2B-C).

\section{EBF1 knockdown inhibit tumor formation in mice}

In order to clarify the effect of EBF1 expression on the proliferation of lung cancer cells in vivo, we injected A549 cells subcutaneously into the back of nude mice. On the 20th day after injection, we found 
that the average tumor weight of A549-shRNA-EBF1 group $(0.37 \pm 0.03 \mathrm{~g})$ was lighter than that of A549shRNA-control group (1.66 $\pm 0.19 \mathrm{~g})$ ((Fig. 3A-B),and EBF1 expression of the tumor decreased significantly in A549-shRNA-EBF1 group (Fig. 3C).

The results showed that the proliferation of $A 549$ cells was significantly inhibited by down-regulating the expression of EBF1, suggesting that inhibiting the expression of EBF1 gene is helpful to inhibit the proliferation of tumor cells in vivo.

4. EBF1 knockdown blocked cell cycle in $\mathrm{G} 1$ phase in A549 cells.

To examine whether knockdown EBF1 inhibit the proliferation of A549 cells was associated with cell cycle arrest, cell cycle distribution was analyzed by flow cytometry (FCM). As shown in Fig.4 , A549 cells with knockdown EBF1 have $41.4 \%$ in the $\mathrm{G} 1$ phase, $39.7 \%$ in the S phase, $14.8 \%$ in $\mathrm{G} 2$ phase; while the control group was only33.1\% in the $\mathrm{G} 1$ phase, $49.1 \%$ in the $\mathrm{S}$ phase, $15.9 \%$ in the $\mathrm{G} 2$ phase, the results showed that knockdown EBF1 induced G0/G1 phase arrest of A549 cells(Fig 4).

5. EBF1 knockdown upregulates the expression of the the tumor suppressor p16 INK4a and cell cycle kinase CDK6

To verify the accurate molecular targets that EBF1 affects the proliferation of lung cancer cells, the protein expressions were evaluated by western blot after knockdown EBF1 expression in A549 cells. The results of the western blot showed that the expression of CDK6 was down-regulated and p21/p27 was increased after knockdown EBF1 in A549 cell, compared with the control group(Fig 5).

\section{Discussion}

Cell cycle can be divided into four stages: G 1, S, G 2 and M, which are closely related to cell proliferation. Abnormal regulation of cell cycle plays an important role in tumorigenesis. Cell cycle progression is regulated by many factors: cyclin, cyclin-dependent kinase (CDK) and cyclin-dependent kinase inhibitor (CDKI). Studies have shown that tumor genes or tumor suppressor genes are associated with cell cycle changes in more than $90 \%$ of human tumors ${ }^{[13]}$. Among them, the frequency of gene changes related to $\mathrm{G}$ 1 phase is higher ${ }^{[14]}$.

Our results showed that EBF1 knockdown in A549 cells could inhibit the proliferation of A549 cells in vitro and in vivo. Generally speaking, cell growth inhibition may be caused by cell necrosis, apoptosis or cell cycle arrest. The results of flow cytometry showed that EBF1-shRNA inhibited the proliferation of A549 cells by blocking the cells in $\mathrm{G} 1$ phase.

Cell cycle is closely related to cell proliferation ${ }^{[15]}$. Upregulation of cyclin expression can lead to uncontrolled cell proliferation and cancer, of which p21/p27 protein ${ }^{[16]}$, which plays a role in $\mathrm{G} 1$ phase, is the most representative ${ }^{[17]}$. Related studies have shown that the decrease of p21 and p27 protein expression will lead to abnormal cell proliferation ${ }^{[18-19] . ~}$ 
Therefore, in order to further explore the mechanism of inhibition of A549 cell proliferation by knockdown EBF1 gene expression, we first detected the expression of p21/p27 protein by western blot, and found that the expression of $\mathrm{p} 21 / \mathrm{p} 27$ protein in A549-shRNA-EBF1 group was up-regulated. What we are interested in is how EBF1 regulates the proliferation of tumor cells such as A549, which is deleted INK4a locus. Kollmann et al[ ${ }^{[20]}$ found that CDK6 promotes proliferation and angiogenesis by up-regulating the expression of vascular endothelial growth factor in the absence of p16 INK4a. Western blot was used to detect the expression of CDK6 protein in A549-shRNA-EBF1 cells. It was found that the expression of CDK6 protein was down-regulated in A549-shRNA-EBF1 group. Of course, whether EBF1 regulates the proliferation of A549 cells by regulating the angiogenesis of vascular endothelial growth factor needs further experiments to confirm.

To summarize, knockdown the expression of EBF1 gene affects the proliferation of A549 cells by blocking A549 cells in G 1 phase, accompanied by an increase in the expression of p21/p27 and a decrease in the expression of CDK6. Therefore, we infer that in A549 cells lacking INK4a/ARF locus, EBF1 regulation of cell cycle is not only related to the increased expression of p21/p27 protein, but also possibly related to angiogenesis through CDK6. However, a large number of experiments are needed to confirm this.

\section{Declarations}

\section{Ethics approval and consent to participate}

All procedures performed in this studythat involved human participants were approved by the Research Ethics Committee of Tianjin Medical University Cancer Institute and Hospital and conformed to the ethical standards of the 1964 Helsinki Declaration.All subjects were over 18 years of age and voluntarily signed informed consent forms.

All the experiments in vivo on the animals in the manuscript that all procedures were carried in accordance with the ARRIVE guidelines. The animal experiments involved in the following project has been reviewed and approved by The Animal Ethical and Welfare Committee of Tianjin Medical University Cancer Institute and Hospital

\section{Consent for publication}

Not applicable

\section{Availability of data and materials}

The datasets used during the current study are available from the corresponding author on reasonable request.

\section{Competing interests}


The authors declare that they have no competing interests

\section{Funding}

Lin WANG :Special fund for laboratory imaging of Tianjin medical university cancer institute and hospital, Y1902

Ding Li: National Natural Science Foundation of China, 81802080

\section{Authors' contributions}

LW Knockdown EBF1 gene in A549 cells for PCR Western blot MTTT $\otimes$ BrdU and animal experiments to detect the effect of EBF1-shRNA on the proliferation of A549 cells in vitro and in vivo and was a major contributor in writing the manuscript.

HF collected lung cancer tissues of all patients.

DL was responsible for data statistics and analysis.

LR designed the entire subject research and reviewed the article.

All authors have read and approved the final manuscript.

\section{Acknowledgements}

Not applicable

\section{References}

1. Yamanashi K, Okumura N, Takahashi A, Nakashima T, Matsuoka T. Surgical and survival outcomes of lung cancer patients with intratumoral lung abscesses.J Cardiothorac Surg. 2017 May 26;12(1):44. doi: 10.1186/s13019-017-0607-3.

2. Rivera GA, Wakelee H.Lung Cancer in Never Smokers.Adv Exp Med Biol. 2016;893:43-57. doi: 10.1007/978-3-319-24223-1_3.

3. O'Leary C, Xu W, Pavlakis N, Richard D, O'Byrne K.Rearranged During Transfection Fusions in NonSmall Cell Lung Cancer.Cancers (Basel). 2019 May 3;11(5). pii: E620. doi: 10.3390/cancers 11050620 .

4. Toyooka S, Shigematsu H, et al. Mutational and epigenetic evidence for independent pathways for lung adenocarcinomas arising in smokers and never smokers [J]. Cancer Res, 2006, 66: 1371-1375.

5. Fragou D, Pakkidi E, Aschner M, Samanidou V, Kovatsi L.Smoking and DNA methylation: Correlation of methylation with smoking behavior and association with diseases and fetus development following prenatal exposure. Food Chem Toxicol. 2019 May 4;129:312-327. doi: 10.1016/j.fct.2019.04.059. 
6. Hu Y, Yoshida T, Georgopoulos K.Transcriptional circuits in B cell transformation.Curr Opin Hematol. 2017 Jul;24(4):345-352. doi: 10.1097/MOH.0000000000000352.

7. Zandi S, Mansson R, Tsapogas P, Zetterblad J, Bryder D, Sigvardsson M. EBF1 is essential for Blineage priming and establishment of a transcription factor network in common lymphoid progenitors. J Immunol. 2008 Sep 1;181(5):3364-72.

8. Melissa L. Holmes, Sebastian Carotta, Lynn M. Corcoran, and Stephen L. Repression of Flt3 by Pax5 is crucial for B-cell lineage commitment. Nutt. Genes Dev. 2006 Apr 15; 20(8): 933-938.doi: $10.1101 /$ gad. 1396206

9. Harvey R. C., Mullighan C. G., Wang X., et al. Identification of novel cluster groups in pediatric highrisk B-precursor acute lymphoblastic leukemia with gene expression profiling: correlation with genome-wide DNA copy number alterations, clinical characteristics, and outcome. Blood. 2010;116(23):4874-4884. doi: 10.1182/blood-2009-08-239681.

10. Schwab C, Ryan SL, Chilton L, Elliott A, Murray J, Richardson S, Wragg C, Moppett J, Cummins M, Tunstall O, Parker CA, Saha V, Goulden N, Vora A,Moorman.A.V,Harrison.C.J.EBF1-PDGFRB fusion in pediatric B-cell precursor acute lymphoblastic leukemia (BCP-ALL): genetic profile and clinical implications. Blood.2016;May,5;127(18):2214-8.doi:10.1182/blood-2015-09-670166.

11. Izraeli S.Beyond Philadelphia: 'Ph-like'B cell precursor acute lymphoblastic leukemias-diagnostic challenges and therapeutic promises. CurrOpinHematol,2014,21(4):289-296.

12. Stone J, Thompson DJ, Dos Santos Silva I, et al.Novel Associations between Common Breast Cancer Susceptibility Variants and Risk-Predicting Mammographic Density Measures. Cancer Res. 2015 Jun 15;75(12):2457-67. doi: 10.1158/0008-5472.CAN-14-2012. Epub 2015 Apr 10.

13. Ko A, Han SY, Song J. Regulatory Network of ARF in Cancer Development. Mol Cells. 2018 May 31;41(5):381-389. doi: 10.14348/molcells.2018.0100. Epub 2018 Apr 18.

14. Icard P, Fournel L, Wu Z, Alifano M, Lincet H. Interconnection between Metabolism and Cell Cycle in Cancer. Trends Biochem Sci. 2019 Jan 14. pii: S0968-0004(18)30273-1. doi: 10.1016/j.tibs.2018.12.007.

15. Caruso JA, Duong MT, Carey JPW, Hunt KK, Keyomarsi K. Low-Molecular-Weight Cyclin E in Human Cancer: Cellular Consequences and Opportunities for Targeted Therapies. Cancer Res. 2018 Oct 1;78(19):5481-5491. doi: 10.1158/0008-5472.

16. Li Y, Huang J, Zeng B, Yang D, Sun J, Yin X, Lu M, Qiu Z, Peng W, Xiang T, Li H, Ren G. PSMD2 regulates breast cancer cell proliferation and cell cycle progression by modulating p21 and p27 proteasomal degradation.Cancer Lett. 2018 Aug 28;430:109-122. doi: 10.1016/j.canlet.2018.05.018. Epub 2018 May 17.

17. Zubair H, Bhardwaj A, Ahmad A, Srivastava SK, Khan MA, Patel GK, Singh S, Singh AP. Hydroxytyrosol Induces Apoptosis and Cell Cycle Arrest and Suppresses Multiple Oncogenic Signaling Pathways in Prostate Cancer Cells. Nutr Cancer. 2017 Aug-Sep;69(6):932-942. doi: 10.1080/01635581.2017.1339818. Epub 2017 Jul 18. 
18. Chang X,Chai Z,Zou J,et al. PADI3 induces cell cycle arrest via the Sirt2/AKT/p21 pathway and acts as a tumor suppressor gene in colon cancer.Cancer Biol Med,2019,16(4): 729-742. doi:10.20892/j.issn.2095-3941.2019.0065.

19. Geng F,Zhang Y,Lu Z,et al. Fusobacterium nucleatum Caused DNA Damage and Promoted Cell Proliferation by the Ku70/p53 Pathway in Oral Cancer Cells.DNA Cell Biol,2020,39(1): 144-151. doi:10.1089/dna.2019.5064.

20. Kollmann K,Heller G,Schneckenleithner C,et al. A Kinase-Independent Function of CDK6 Links the Cell Cycle to Tumor Angiogenesis.Cancer Cell,2016,30(2): 359-360. doi: 10.1016/j.ccell.2016.07.003.

\section{Figures}

A

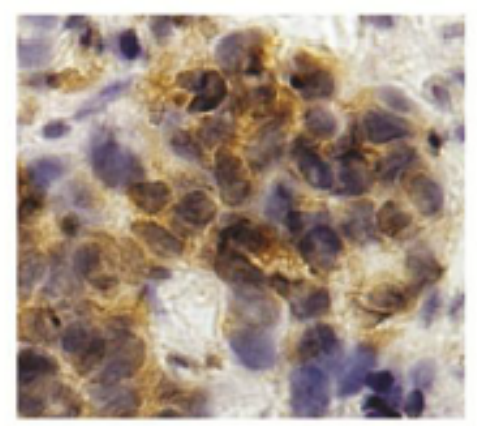

NSCLC

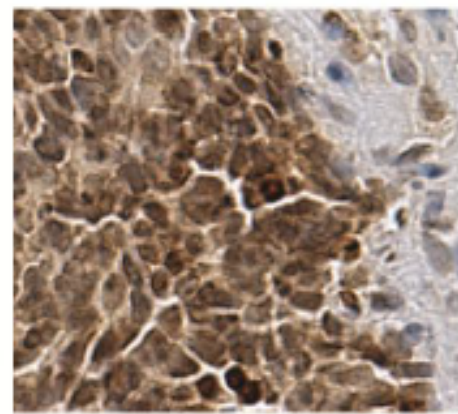

SCLC

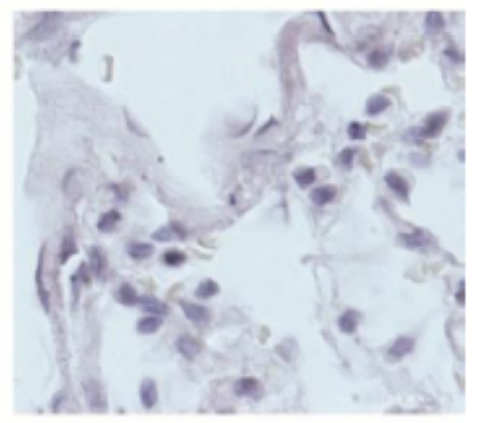

Peripheral Lung Tissue

B
A549
H441
H209
H1155
HBE
Beas-2B

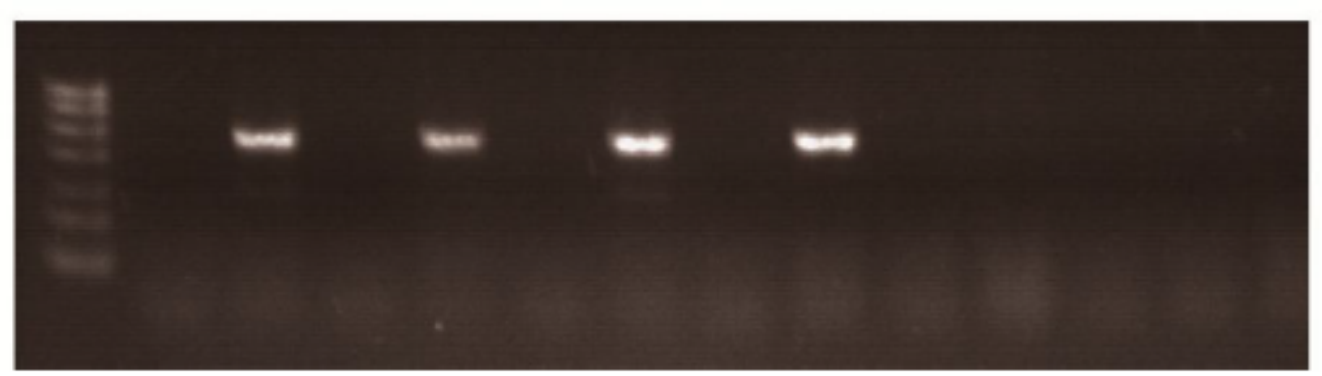

\section{Figure 1}

A: IHC staining with anti-EBF1 antibody show positive EBF1 staining in lung cancer: EBF1 staining in NSCLC cells ; and SCLC cells ; lack of EBF1 staining in normal lung epithelial cells. B: RT-PCR analysis of EBF1 expression in various cell lines. 

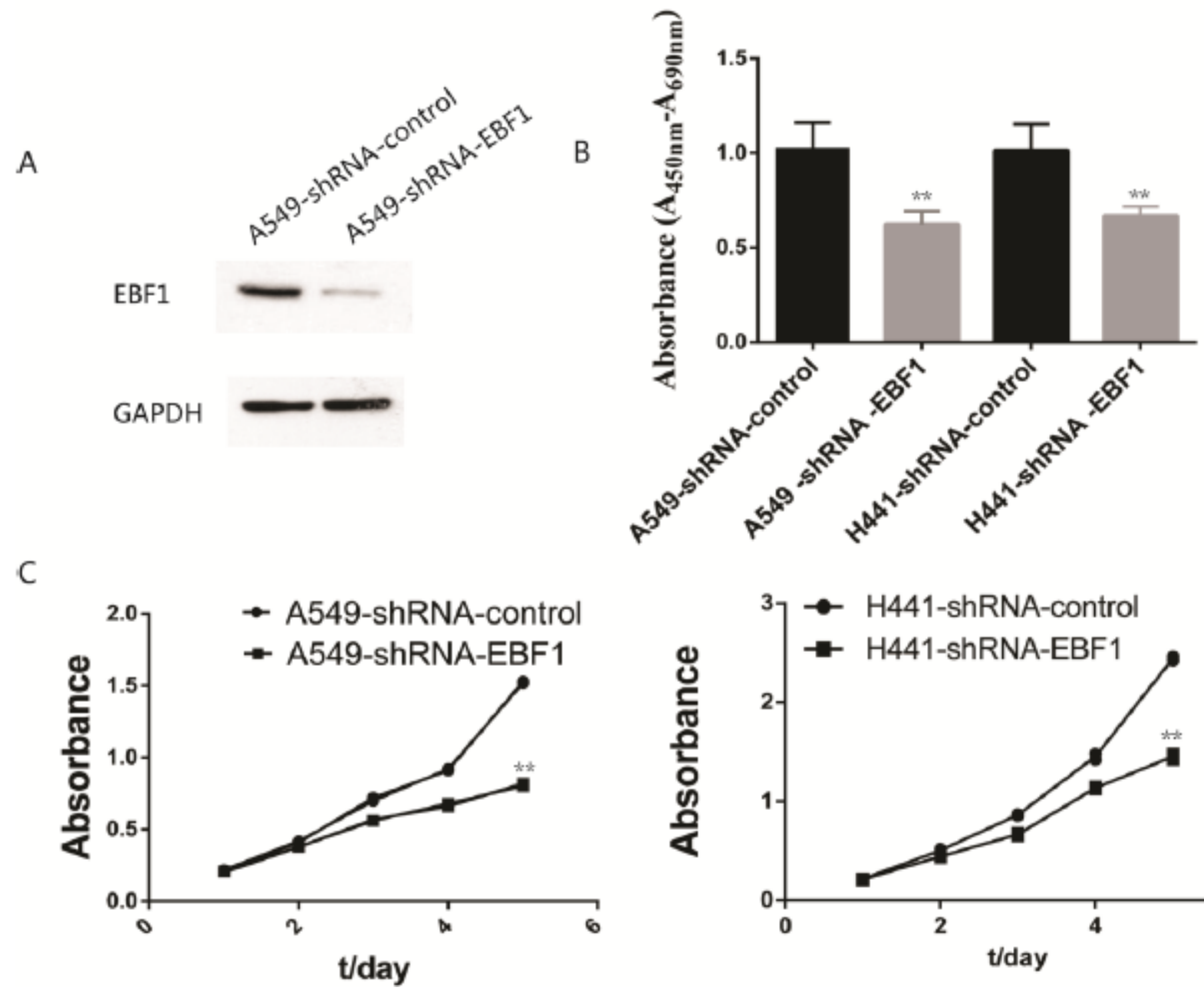

Figure 2

EBF1 knockdown inhibit cell proliferation in A549 cells A: Western blot shows that the expression of EBF1 in A549 cells is decreased by transfected with specific shRNA; B: Analysis of cell proliferation using BrdU experiment in A549 and H441 cells, $* * P<0.05$; C: Analysis of cell viability using the MTT assay in A549 and $\mathrm{H} 441$ cells for 5 days, ${ }^{\star *} \mathrm{P}<0.05$ 
A

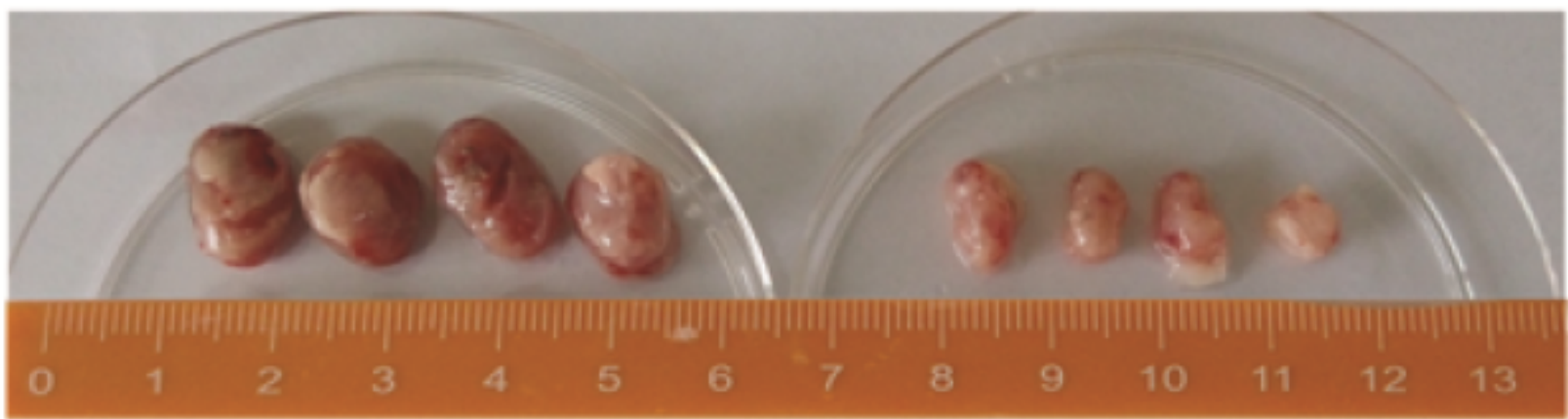

A549-shRNA-cntrol

B

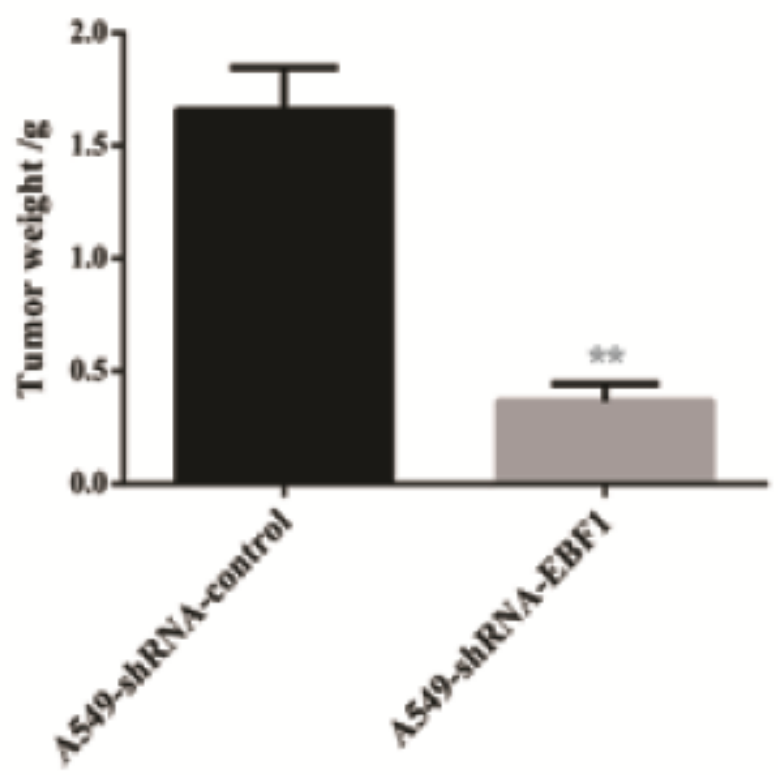

\section{A549-shRNA-EBF1}

C

A 549-shRNA-cntrol

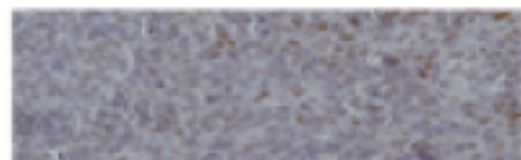

A S49-shRNA-EBF1

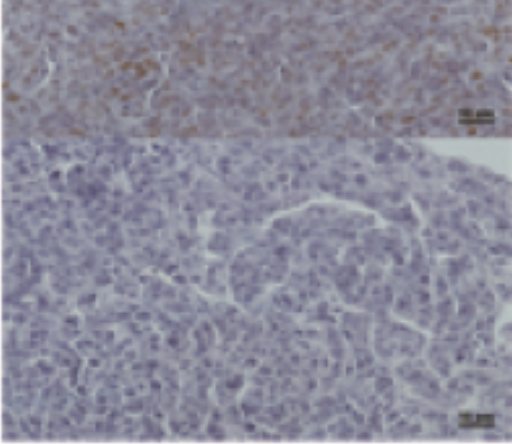

\section{Figure 3}

A: Animal experiments showed that EBF1 knockdown inhibit tumor formation in vivo; B: Average weight of tumors, ${ }^{\star \star P}<<0.05$; $\mathrm{C}$ : EBF1 expression of the tumor decreased after EBF1 knockdown. 


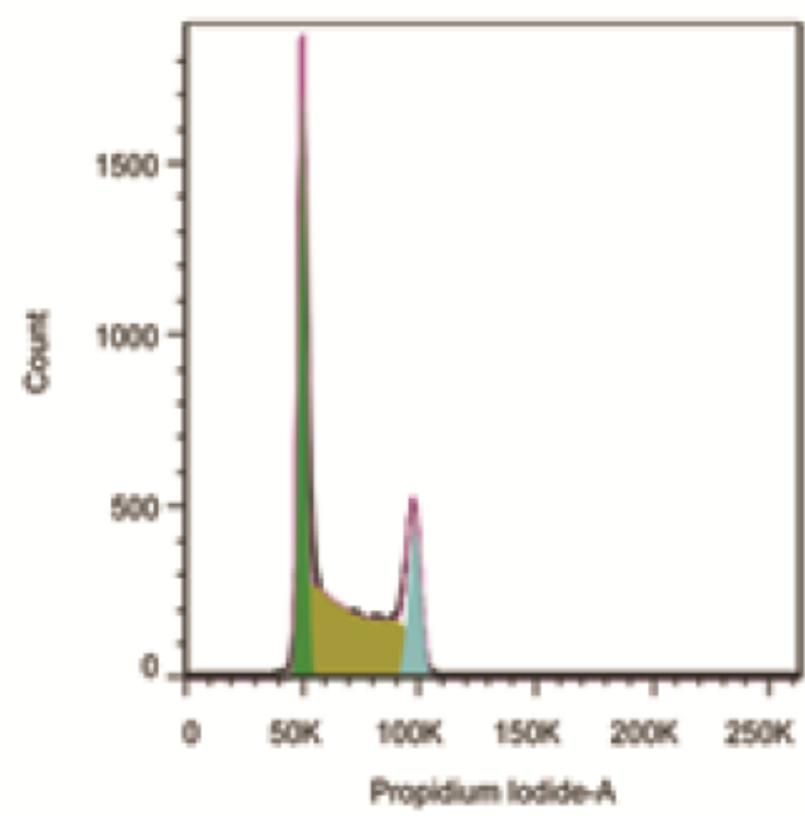

A549-shRNA-control

Freq.G1 $=33.06$

Freqs $=49.06$

Freq. $62=15.89$

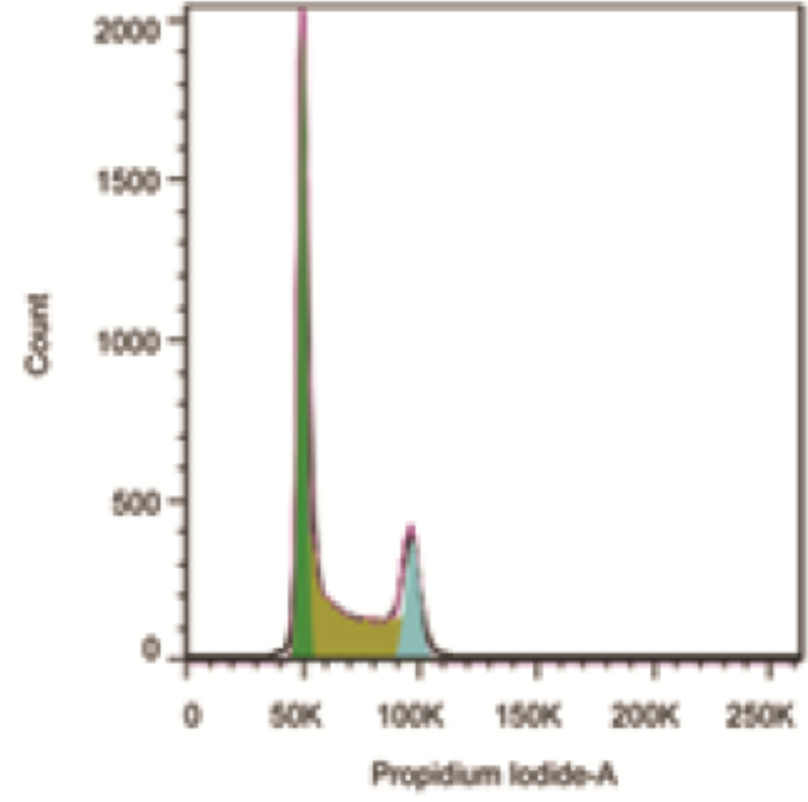

A549-AhRNA-FBFI

Freq, $G 1=41.41$

Freq. $\mathrm{S}=39.73$

Freq, $62=14,78$

Figure 4

EBF1 knockdown affects cell cycle of A549 cells 


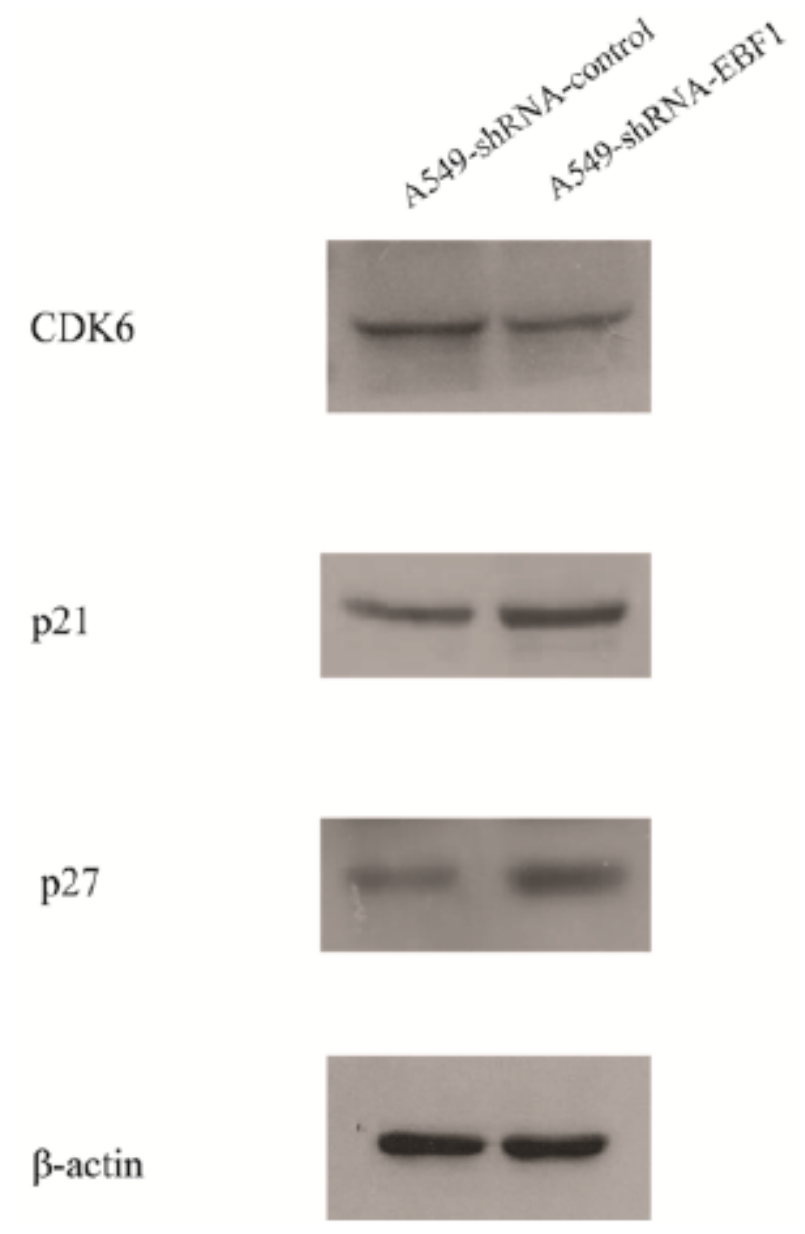

\section{Figure 5}

EBF1 knockdown affects cyclin expression of A549 cells 